\title{
Epilepsy and the Autisms
}

\section{Mary Coleman*}

Foundation of Autism Research, USA

\section{Editorial}

Epilepsy and autism have a strong association. The hypothesis of excitatory/inhibitory imbalance first developed in epilepsy is now under serious consideration in autism. Such a hypothesis might help explain why $30 \%$ of individuals with one of The Autisms, many of them not yet diagnosed with their underlying disease entity, have a seizure disorder; this is a figure higher than is found even in severely retarded individuals. There are at least 35 diagnosed disease entities with autism/ epilepsy described in the medical literature [1] and now that the cost of genome sequencing has dropped to an affordable range, undoubtedly many more will be identified.

Epilepsy and autism share other characteristics. Neither is a disease entity in itself, but rather the final common expression of dysfunctional brain networks of many different underlying disease entities. However, epilepsy and autism are different in the respect in that epilepsy is episodic, while autistic features are not. But it should be noted that one study of 899 individuals with autism found that $61 \%$ of them had epileptiform activity during sleep, even though they did not have clinical seizures [2]. Generally the cognitive level is lower is those individuals who have autism/epilepsy.

Many patterns of seizures are seen in patients with one of The Autisms - infantile spasms, atonic seizures, myoclonic seizures, atypical absence (petit mal), and complex partial seizures and generalized tonicclonic. Infantile spasms and complex partial seizures are relatively more common than other seizures types in this patient group.

Treatment with anti-epileptic drugs (AEDs) aims to reduce or stop seizures in most individuals with one of The Autisms and no change in autistic features is expected. However there are a few exceptions where the clear features of autism recede with intensive anti-seizure treatment.

\section{These exceptions are:}

In the Landau-Kleffner syndrome, autistic features may remit with medical or surgical treatment

Early onset lesional epilepsy, particularly dysembryoplastic temporal lobe lesions showing autistic regression, may remit with early effective surgery [3].

The advent of whole exome/genome sequencing and the technology-driven reduction in the cost of next generation sequencing, as well as the introduction of diagnostic targeted sequencing chips, have resulted in an unprecedented volume of data directly linking patient genomic variability to disorders of the brain. Both epilepsy and autism already have and will continue to greatly benefit from these developments. We are entering a new age of medicine where disease entities may be known by their genomic mutations as much as by their clinical symptoms.

Looking to the future, for many individuals the ideal way to control seizure activity will to correct the underlying genomic error causing the symptoms, eliminating both the seizures and sometimes, also the concomitant autistic symptoms. An example of this approach is seen one of the diseases with autism/epilepsy -- the tuberous sclerosis complex, caused by mutations in the genes TSC1 or TSC2 that abnormally activate the mammalian target of rapamycin (mTOR). Under normal cellular conditions, the mammalian target of rapamycin (mTOR) regulates cell growth and proliferation in response to signals from nutrients or growth factors, but loss of TSC1 or TSC2 leads to overactivation of mTOR and uncontrolled cellular proliferation. Everolimus is an mTOR inhibitor approved for use in a number of indications where mTOR over activation is implicated, including the tuberous sclerosis complex [4].

This is a fascinating time to be reevaluating patients with autism/ epilepsy and looking for new approaches to successful medial therapy. It is anticipated that new genomic understanding, both genetic and epigenetic, of the mechanisms of action underlying seizures currently not successfully controlled in autism/epilepsy can be eventually deciphered and lead to better or excellent control.

\section{References}

1. Coleman M, Gillberg C (2012) The Autisms. Oxford, USA: 351.

2. Chez MG, Chang M, Krasne V, Coughlan C, Kominsky M, et al. (2006) Frequency of epileptiform EEG abnormalities in a sequential screening of autistic patients with no known clinical epilepsy from 1996 to 2005. Epilepsy Behav 8: 267-271.

3. Neville BG, Harkness WF, Cross JH, Cass HC, Burch VC, et al. (1997) Surgical treatment of severe autistic regression in childhood epilepsy. Pediatr Neurol 16: $137-140$.

4. Tran LH, Zupanc ML (2015) Long-Term Everolimus Treatment in Individuals With Tuberous Sclerosis Complex: A Review of the Current Literature. Pediatr Neurol 53: 23-30.
*Corresponding author: Mary Coleman, Director, Foundation of Autism Research, USA, E-mail: mary.coleman.md@gmail.com

Received September 15, 2015; Accepted September 16, 2015; Published September 24, 2015

Citation: Coleman M (2015) Epilepsy and the Autisms. J Ergonomics 1: e101. doi:10.4172/2472-0895.1000e101

Copyright: (c) 2015 Coleman M. This is an open-access article distributed under the terms of the Creative Commons Attribution License, which permits unrestricted use, distribution, and reproduction in any medium, provided the original author and source are credited. 